\title{
Une « nouvelle offensive »
}

L'évolution du système éducatif en Allemagne

Reforming Germany's education system - the new battlefront

Una «nueva ofensiva». La evolución del sistema educativo en Alemania

\section{Ingrid Gogolin}

Traducteur : Dominique Gélin

\section{OpenEdition}

\section{Journals}

Édition électronique

URL : https://journals.openedition.org/ries/2413

DOI : 10.4000/ries.2413

ISSN : 2261-4265

Éditeur

France Education international

Édition imprimée

Date de publication : 1 décembre 2000

Pagination : 29-39

ISSN : 1254-4590

\section{Référence électronique}

Ingrid Gogolin, « Une « nouvelle offensive » », Revue internationale d'éducation de Sèvres [En ligne], 28 | 2000, mis en ligne le 01 décembre 2003, consulté le 09 juillet 2021. URL : http:// journals.openedition.org/ries/2413; DOI : https://doi.org/10.4000/ries.2413 


\title{
Une " nouvelle offensive "
}

\section{L'évolution du système éducatif en Allemagne ${ }^{1}$}

\author{
Ingrid Gogolin
}

\section{Résumé}

L'amélioration des résultats scolaires occupe une place centrale dans les débats sur l'éducation en Allemagne, notamment au vu des conclusions de récentes enquêtes internationales. Cette question est analysée ici dans une double perspective, celle des établissements (quel mode de financement?) et celle des élèves (comment prendre en compte le pluralisme des langues et des cultures?).

Reforming Germany's education system - the new battlefront

The issue of how to improve education standards is one of the central concerns for educational policy in Germany, particularly given the country's scores in recent international studies. The author examines this question from a twofold perspective: Which forms of financing for schools? What strategies to adopt in dealing with linguistic and cultural diversity?

Una " nueva ofensiva ». La evolución del sistema educativo en Alemania El mejoramiento de los resultados escolares es el tema principal de los debates sobre la educación en Alemania, en particular tras los resultados de recientes encuestas internacionales. Esta cuestión se analiza aquí bajo una doble perspectiva, la de los establecimientos (¿Qué modo de financiación?) y la de los alumnos (¿Cómo tomar en cuenta la pluralidad de idiomas y culturas?).

1 Cet article a été traduit par Dominique Gelin. 
Si l'on suit de près les débats sur l'éducation en Allemagne durant les dernières années du Xxe siècle, on a le sentiment qu'une volonté de réforme de grande envergure est à l'œuvre. Il semblerait que les profonds changements culturels, sociaux et techniques des dernières décennies aient poussé à réfléchir sur les contenus, les structures et les modèles d'organisation du système scolaire allemand, après que certains doutes se soient exprimés quant à sa qualité. Les mauvaises performances économiques du pays ont contribué à accentuer le phénomène avec, pour conséquence, l'idée insidieuse que le financement du système scolaire sur les deniers publics ne pouvait plus aller de soi. On prévoit, sur le plan économique, de choisir de nouvelles orientations, dans le but avoué de ne pas remettre en question la qualité du système scolaire, à une époque où les moyens financiers se raréfient. Tout ceci dans un climat où il est beaucoup question, officiellement, d'une "nouvelle offensive" dans le domaine de l'éducation. Un chef d'État comme Johannes Rau en est un bon exemple, parmi d'autres responsables politiques, lui qui a tenu, en l'an 2000, un grand discours sur l'importance croissante qu'il fallait accorder à une excellente éducation des générations futures. On insiste sur l'importance considérable qu'il faut attribuer à l'enseignement en tant que matière première pour permettre à l'Allemagne d'avoir quelque chance de continuer à tenir un rôle prédominant au sein de la concurrence internationale.

Sans chercher à dresser un tableau complet de toutes les évolutions qui vont dans le sens de cette volonté de réforme et qui ont pu être observées récemment dans la République fédérale allemande, deux points seront brièvement abordés et le troisième le sera de façon un peu plus détaillée, dans la mesure où il illustre parfaitement les évolutions en question. La participation croissante de l'Allemagne aux comparaisons internationales des résultats scolaires et les nouvelles tendances de l'évolution du système scolaire en fonction des impératifs économiques seront évoqués rapidement. On présentera de manière plus détaillée les tentatives de réforme qui répondent à la diversité croissante des langues et des cultures de la communauté scolaire en Allemagne.

\section{Comparaisons internationales des résultats scolaires}

L'Allemagne a pris part aux études internationales portant sur la comparaison des résultats scolaires depuis la fin des années soixante, à l'International Study of Reading Literacy par exemple, dont les conclusions ont été publiées au milieu des années quatre-vingt-dix. Ces travaux n'ont cependant pas trouvé beaucoup d'écho sur la place publique. Bien que l'Allemagne ait toujours figuré dans la partie médiane ou inférieure de l'échelle, personne n'a pris acte de ces études dans le pays. Elles n'ont fait l'objet d'un débat que dans le cercle scientifique, dont la spécialité était précisément les sciences comparatives de l'éducation au niveau international. Les choses ont changé, d'une 
manière radicale, lorsque l'Allemagne a participé au Third International Mathematics and Science Study (TIMSS), dont les conclusions ont été publiées à partir de 1998. Depuis, il règne la plus grande effervescence au sujet de ces études dont on explore le sens et l'utilité. Le système éducatif s'est senti concerné à tous les niveaux. Le TIMSS a été à l'origine de grandes controverses parmi les scientifiques, suscitant des travaux de recherche plus larges. Il a eu des conséquences tant dans le domaine de la politique éducative que dans celui des pratiques scolaires ${ }^{2}$. À la suite du TIMSS, une participation fébrile aux études comparatives internationales s'est mise en place, à laquelle s'est ajouté un vaste mouvement d'études comparatives nationales qui ont été menées, pour une part, à l'intérieur de chaque Bundesland et, pour une autre, entre les différents Länder. L'Allemagne participe à l'heure actuelle aux études internationales suivantes : CivEd (Civic Education Study; collecte des données : 1999, conclusions : 2001), PISA (Programme for International Student Assessment; collecte des données : 2000, 2003, 2006, premières conclusions : fin 2001) et PIRLS (Progress in International Reading Literacy Study; collecte des données : 2001, conclusions : 2003). À cela s'ajoutent trois études à grande échelle où sont comparés les résultats scolaires des Bundesländer et cinq autres qui se déroulent à l'intérieur de ces mêmes Länder.

Force est de constater qu'au cours des dernières années du XXe siècle une sorte de fièvre comparative s'est emparée du système éducatif allemand. Cette tendance, rationnelle en partie seulement, est également à mettre au compte des phénomènes de modes qui interviennent de façon cyclique dans le paysage de la politique éducative et de la recherche, en tenant compte toutefois $\mathrm{du}$ fait que la tendance générale à l'internationalisation et à la globalisation trouve sa résonance au niveau scientifique et politique national. Le sens et l'utilité de ces enquêtes à grande échelle font l'objet d'une vive controverse en Allemagne autour de deux pôles extrêmes.

D'un côté - celui des partisans de telles études -, on place de grands espoirs dans ces travaux dans la mesure où ils permettent, entre autres, de comparer les normes en vigueur pour évaluer les résultats des divers cursus dans les écoles allemandes. Le système éducatif allemand se caractérise, traditionnellement, par sa grande ouverture : en effet, le pouvoir de décision ne se situe pas, en ce qui le concerne, au niveau du gouvernement fédéral mais au niveau des différents Länder; en outre, il n'y a pas de système général d'examens centralisés. Les Länder garantissent aux élèves la reconnaissance réciproque des diplômes de fin d'études, bien que les types d'établissements scolaires existants ne soient pas toujours les mêmes dans chacun d'eux. Les partisans de ces études caressent en particulier l'espoir que la comparaison des résultats scolaires

2 G. Behler, "Auf dem Weg zu mehr Qualität und Vergleichbarkeit », Zeitschrift für Erziehungswissenschaft, $n^{\circ}$, 1999, p. 423-430. 
permette de comparer avec une plus grande fiabilité les diplômes de fin d'études, sans qu'il soit nécessaire de recourir à une autre forme d'organisation du système scolaire, plus fortement centralisée ${ }^{3}$. D'autres espoirs portent sur l'évolution de l'école. Faire redescendre les conclusions des études comparatives jusqu'au niveau des établissements qui y ont participé, devrait les conforter, diton, dans leur effort d'amélioration de la qualité de leur travail ${ }^{4}$.

On entend, de l'autre côté, un grand nombre de critiques, mettant en garde contre une surestimation de ce qu'on pourrait en attendre tant dans la mise au point de normes appropriées que dans l'évolution de l'école. Les mises en garde s'appuient sur certaines expériences dont on dispose aux États-Unis : il est apparu que la grande quantité des évaluations externes a eu pour effet de rendre les écoles où il y avait une volonté de faire avancer les choses sur le plan de la pédagogie de façon active et constructive, incapables de les réaliser, les paralysant pratiquement ${ }^{5}$. Les représentants des syndicats critiquent de manière particulièrement opiniâtre l'euphorie des tests en Allemagne ${ }^{6}$. Ici s'exprime la crainte que l'emploi de ces outils ait pour conséquence non pas l'amélioration du système d'enseignement, mais bien au contraire une aggravation de la situation, que personne ne souhaite. On estime, en particulier, qu'il est tout à fait déplorable de réduire tant les résultats des élèves que ceux des écoles uniquement à quelque chose de mesurable, de faire seulement appel à des paramètres chiffrables, sans nuances. Ceci ne permettrait de rendre compte, de façon équitable, ni de la complexité du travail scolaire ni de ce qu'on revendique : un processus de formation qui devrait, au-delà du contrôle du savoir, développer des compétences qu'il n'est pas possible d'attester à l'aide de tests d'évaluation (savoir communiquer, compétences dans les domaines sociaux et esthétiques).

\section{La soumission du système éducatif aux impératifs économiques}

Il existe une étroite relation entre ce qui se passe dans le domaine de la comparaison des résultats scolaires et le second débat, qui va servir d'exemple ici, portant sur une modification du financement de l'éducation en Allemagne.

3 0. Köller, J. Baumert, K. U. Schnabel, «Wege zur Hochschulreife : Offenheit des Systems und Sicherung vergleichbarer Standards », Zeitschrift für Erziehungswissenschaft, n 3, 1999, p. 385-422.

4 R. Lehmann (et al.), Aspekte der Lernausgangslage und der Lernentwicklung von Schülerinnen und Schülern an Hamburger Schulen (LAU), Hamburg, 1999, également consultable : http://www.lbs.hh.schule.de/lau/lau7/

5 G. Kempfert, H.-G. Rolff, Pädagogische Qualitätsentwicklung, Weinheim, 1999 ; Ch. Klug, S. Reh, « Was fangen die Schulen mit den Ergebnissen an? Die Hamburger Leistungsvergleiche aus der Sicht "beforschter" Schulen ", Pädagogik, $n^{\circ} 12,2000$, p. 16-21.

6 M. Demmer, "Risiken und Nebenwirkungen von Schulleistungsvergleichen. Argumente gegen die Testeuphorie ", Pädagogik, $n^{\circ}$ 12, 2000, p. 32-35. 
Il est certain que la situation de plus en plus tendue du budget public allemand a, tout d'abord, été à l'origine de cette question, mais ceci est allé de pair avec une critique croissante des performances du système éducatif, dont le niveau insuffisant avait été démontré par les résultats très moyens obtenus lors des comparaisons internationales. Dans l'esprit de ceux qui émettent ces critiques, l'offre d'enseignement ne correspond pas assez à la demande des utilisateurs. Le système éducatif ne réagirait pas suffisamment aux exigences de ses "clients » indirects - c'est-à-dire les employeurs et l'économie - et ne répondrait pas non plus aux besoins de sa " clientèle » directe : les élèves. On considère, de plusieurs côtés, qu'il faut lui donner une orientation plus conforme aux exigences du marché pour améliorer cet état des choses, d'une part, en introduisant des mécanismes de concurrence entre les écoles et, d'autre part, en ayant recours aux méthodes de financement et de prise de décision utilisées dans le secteur de l'économie.

Historiquement, l'idée de faire jouer la concurrence dans le système éducatif n'est pas un point de vue très répandu dans le système éducatif allemand. On a, certes, mis en place un système scolaire à première vue bien différencié : dans la plupart des Länder, après un parcours commun de quatre ans à l'école élémentaire (six ans dans certains Länder), les élèves, filles et garçons, sont orientés vers des établissements scolaires de différentes natures, qui doivent correspondre aux différents types de résultats scolaires. Il y a en principe trois voies, aboutissant à trois types de qualifications différents. Les écoles du type Hauptschule s'adressent à des élèves dont les compétences de type cognitif sont moins développées mais qui, par contre, ont davantage de dispositions pratiques. Elles délivrent, d'un point de vue formel, le diplôme de fin d'études le moins élevé. Les écoles du type Realschule où les élèves doivent faire preuve de compétences cognitives un peu plus élevées, tout en ayant les mêmes dispositions pratiques, délivrent un diplôme de fin d'études de niveau intermédiaire. Enfin, les lycées (Gymnasium) accueillent les élèves ayant les meilleures compétences de type cognitif et mènent au diplôme de fin d'études secondaires le plus élevé (Abitur). Depuis les années quatre-vingt le nombre des élèves scolarisables est en baisse et on voit, de plus en plus, les différents types d'écoles entrer en concurrence pour recruter des élèves. Les parents qui attachent du prix à l'ascension sociale et au rôle que l'éducation peut y jouer tiennent beaucoup à ce que leurs enfants obtiennent l'Abitur et environ un tiers des élèves qui quittent le système scolaire obtiennent ce diplôme en Allemagne. Mais, en fait, la concurrence entre les écoles est plutôt absente du système éducatif allemand. Cela tient en partie à ce qu'il n'y a pratiquement pas d'écoles privées : à peine $5 \%$ de la totalité des élèves fréquentent des établissements privés d'enseignement général ${ }^{7}$.

7 G. Bellenberg, W. Böttcher, K. Klemm, "Schule und Unterricht ", in : W. Böttcher, K. Klemm, Th. Rauschenbach, Bildung und Soziales in Zahlen, Weinheim, Munich, Juventa, 2001, p. 93-126. 
Ceux qui sont partisans d'adopter dans le système éducatif ( $\mathrm{y}$ compris, du reste, dans le troisième cycle, c'est-à-dire dans les universités et les instituts universitaires, dont il ne sera pas davantage question ici) les modes de fonctionnement en usage dans l'économie, avancent l'argument qu'introduire de tels modes de financement, conformes aux exigences du marché, permettrait d'obtenir un meilleur équilibre entre l'offre et la demande en matière d'éducation. Il faudrait, dans cette optique, que l'État assure le financement de base, complété par un système de primes accordées en fonction des résultats obtenus. Les écoles particulièrement performantes qui offriraient à leurs élèves la meilleure garantie de réussite devraient, grâce à leur profil, bénéficier de moyens supplémentaires afin de pouvoir continuer à développer leurs compétences. Davantage d'autonomie : c'est le mot clé en vertu duquel les écoles se verraient de plus en plus autorisées à gérer leur propre budget. Cela devrait petit à petit entraîner la disparition des procédés habituels de gestion centralisée des moyens financiers par l'État et, dans la même logique, l'acquisition de moyens supplémentaires issus du privé et utilisés dans le budget de l'école. Cela devrait également avoir pour conséquence une relative diminution du contrôle des écoles par l'État, leur donnant la possibilité de répondre plus rapidement, et de façon plus adéquate, aux vœux de leur «clientèle ${ }^{8}$.

Les partisans de cette évolution avancent aussi l'argument que chaque école aurait plus de chance de se doter d'un profil attractif, bien adapté aux élèves qui s'y inscrivent. Grâce à ce système attribuant des moyens matériels supplémentaires en fonction des performances, les enseignants seraient davantage prêts à s'investir dans une amélioration de la qualité de leur travail et les équipes de direction seraient plus motivées.

Ce sont précisément, du point de vue de ceux qui critiquent l'introduction dans le système éducatif de tels modes de financement, ces effets prétendument si positifs qui posent problème. Les études sur les systèmes éducatifs, où de telles dispositions ont été prises, ont mis en évidence, disent-ils, des risques considérables : en premier lieu, celui de susciter des inégalités entre les écoles fréquentées par des élèves issus des couches défavorisées de la société - défavorisées à la fois sur le plan économique et sur le plan éducatif - et les autres établissements. Ces écoles ne sont guère, selon eux, en mesure de profiter des modes de fonctionnement en usage dans l'économie. Qu'il s'agisse de l'équipement matériel ou des performances au niveau des résultats scolaires, l'écart qui existe traditionnellement entre ces écoles et celles fréquentées par des élèves issus de couches plus favorisées de la société aurait tendance à se creuser à une vitesse vertigineuse ${ }^{9}$. Et, par là même, le principe fondamental selon lequel le système éducatif allemand garantit une égalité des chances à tous les élèves serait menacé. Accorder de plus

8 W. Böttcher, P. Weishaupt, W. Weiss (éd.), Wege zu einer neuen Bildungsökonomie, Weinheim et Munich, 1997.

9 R. Hatcher, "Labour, Official School Improvement and Equality ", Journal of Education Policy, n 4, 1998, p. $485-499$. 
en plus d'importance aux paramètres économiques et appliquer de tels modes de fonctionnement remettrait en question, selon ces critiques, la mission même du système éducatif allemand qui est, fondamentalement, de prendre en charge toute la population scolaire. Cela reviendrait à abandonner la voie choisie, dans la perspective du libéralisme bourgeois classique, d'un modèle de société dont les institutions scolaires publiques, instaurées par la communauté pour la communauté, sont un élément essentiel. La conséquence étant qu'on se tournerait vers un système néoliberal où le domaine social en général ferait l'objet d'une commercialisation, à laquelle l'école publique - parmi d'autres concepts comme, par exemple, la " liberté » dont doivent jouir les sciences - serait sacrifiée sans qu'on se pose davantage de questions d'ordre éthique ${ }^{10}$.

\section{Pluralisme des langues et des cultures}

Nous allons à présent étudier de façon un peu plus détaillée les débats suscités par le troisième sujet, c'est-à-dire la question de savoir comment le système éducatif allemand, en tant qu'institution étatique nationale, doit réagir face au pluralisme de plus en plus prégnant des langues et des cultures dans la population scolaire, du fait, entre autres, des migrations internationales de la deuxième moitié du siècle écoulé.

Il existe au moins deux points communs entre ces débats et ceux abordés ci-dessus.

En premier lieu, il ressort constamment des études comparatives nationales et internationales que les élèves originaires de familles immigrées obtiennent des résultats scolaires inférieurs à ceux des élèves allemands. Les enfants qui détiennent un passeport allemand et ceux qui n'en ont pas, n'ont donc pas les mêmes chances de réussite dans le système scolaire allemand. Ces conclusions viennent contredire le principe fondamental, allant de soi dans tout système éducatif, selon lequel l'égalité des chances doit être garantie à tous les élèves. Conclusions que viennent étayer les statistiques portant sur la fréquentation de l'ensemble des établissements scolaires en Allemagne : les élèves d'origine étrangère sont, sans qu'on puisse constater aucune évolution, presque deux fois plus nombreux que les élèves allemands à fréquenter les Hauptschulen, écoles qui offrent, comme on l'a vu, le diplôme de fin d'études le moins élevé. Ils sont, par contre, deux fois moins nombreux que les élèves allemands à fréquenter les lycées (Gymnasium), établissements qui permettent d'obtenir le diplôme le plus prestigieux ${ }^{11}$.

10 I. Lohmann, "Multimediale Mehrwertproduktion. Über die Kommerzialiesierung von Informationen und Bildung », Forum Wissenschaft, $n^{\circ} 4,1999$, p. 9-14.

11 Y. Karaka_O_Ly-Aydin, "Kinder aus Zuwandererfamilien im Bildungswesen ", in : W. Böttcher, K. Klemm, Th. Rauschenbach, Bildung und Soziales in Zahlen, Weinheim, Munich, 2001, p. 273-302. 
En outre, les études comparatives internationales concernant les écoles que l'on avait assujetties aux impératifs économiques ont montré quelles conséquences néfastes cela pouvait avoir sur les établissements fréquentés par un pourcentage important d'élèves originaires de familles immigrées. Ces écoles se retrouvent du côté de ceux qui ont tout à perdre lorsque de telles réformes sont appliquées ${ }^{12}$.

Ceci constitue le cadre dans lequel une intense activité de recherche s'est développée afin de tenter de trouver les raisons pour lesquelles le système éducatif allemand était ainsi dans l'incapacité de garantir aux enfants originaires de familles immigrées une égalité des chances ${ }^{13}$. Les conclusions de l'une de ces recherches qui portait, dans ce contexte, sur les réactions des écoles des seize Länder à l'immigration vont être abordées maintenant ${ }^{14}$.

Il faut souligner qu'il y a de grandes différences, d'une région à l'autre, dans le nombre et le pourcentage d'habitants d'origine étrangère dans la République fédérale allemande. Elles sont, certes, particulièrement marquées entre les Länder situés à l'Ouest et ceux situés à l'Est de l'Allemagne. Dans le Brandebourg, le pourcentage d'habitants d'origine étrangère ne dépassait pas jusqu'ici $2,5 \%$ de l'ensemble de la population, tandis que, dans tous les autres Länder de l'Ouest de l'Allemagne, il s'élève à environ $10 \%$ (dans une fourchette allant d'environ $5 \%$ au Schleswig-Holstein à plus de $16 \%$ à Hambourg). Mais ces différences sont également importantes si l'on compare les Länder ouestallemands entre eux. C'est dans les grandes conurbations que l'on trouve traditionnellement les pourcentages les plus élevés : dans les années quatre-vingt-dix, si l'on considère l'ensemble des habitants d'origine étrangère, plus de $60 \%$ vivaient en zones urbaines ${ }^{15}$. Parallèlement, les enfants originaires de familles immigrées se repartissent de manière différente selon les Länder. Globalement, leur nombre n'a cessé de croître, depuis les années cinquante, dans. la République fédérale allemande, aussi bien en ce qui concerne l'enseignement général que l'enseignement professionnel. Il s'élève, entre-temps, à plus de 1,12 million (environ $10 \%$ de l'ensemble de la population scolaire). Il existe, là aussi, des différences considérables entre les Länder, les pourcentages allant d'environ $20 \%$ à Hambourg à moins de $2,5 \%$ dans les Länder est-allemands. À Francfort, ville où l'on trouve le plus fort pourcentage d'habitants d'origine étrangère, environ un tiers de la population n'est pas d'origine allemande.

12 R. Hatcher, "The Politics of School Effectiveness and Improvement ", Race, Ethnicity and Education, $n^{\circ} 2$, 1998, p. 267-289.

13 I. Gogolin, B. Nauck (éd), Migration, gesellschaftliche Differenzierung und Bildung, Opladen, 2000.

14 I. Gogolin, U. Neumann, L. Reuter (éd.), Schulbildung für Kinder von Minderheiten in der BRD - 1989 bis 1999 , Munster, New York, 2000.

15 R. Münz, W. Seifert, R. Ulrich, Zuwanderung nach Deutschland. Strukturen, Wirkungen, Perspektiven, Frankfurt, New York, 1997. 
L'étude présentée ici devait répondre à la question suivante : les migrations ont-elles entrainé des réformes dans le système éducatif d'une Allemagne devenue de facto un pays d'immigration ? ${ }^{16}$ Elle partait du fait établi que le système éducatif allemand avait toujours réagi, en présence des minorités, soit en les isolant, soit en les traitant comme un phénomène particulier. On avait pris des dispositions spécifiques au niveau de l'organisation des établissements, des cursus, sur le plan didactique, au niveau des méthodes d'enseignement, pour répondre aux besoins particuliers de ces minorités. Ce qui, en même temps, permettait d'assurer la stabilité d'un système éducatif valable pour tous ${ }^{17}$. Ce qui nous intéressait était de savoir si les écoles allemandes maîtrisaient toujours de la même façon les migrations, devenues des phénomènes de masse, ou bien si l'on pouvait voir s'esquisser des mouvements de réformes dont on trouverait des répercussions jusque dans le système scolaire "régulier ".

La partie de l'enseignement général consacrée à la maîtrise de la langue, qui sera présentée ici, constitue un exemple caractéristique de la façon dont des pratiques innovantes sont passées dans le système scolaire à partir des problèmes que posaient les migrations. La question de savoir quels jugements on pouvait porter sur les réformes concernant la maîtrise de la langue en cours dans les systèmes scolaires des différents Länder, revêtait une importance toute particulière et devait être prise en considération pour définir le principe directeur de l'étude sur le plan théorique. Parmi les convictions profondes liées à la maîtrise de la langue dans le système éducatif général, celle d'un système éducatif au service de l'État et de la nation ressort particulièrement. Certes, enseigner l'allemand dans une école allemande, en allemand, est le reflet d'une attitude pragmatique : on accorde la priorité à la langue de communication locale. Mais on voit aussi s'y exprimer, le principe d'un monolinguisme - héritage historique - qu'on trouve dans le système éducatif allemand comme dans d'autres systèmes éducatifs eux aussi au service, de façon très classique, de l'État et de la nation. Des signes indiquant que cet obstacle est surmonté constituent, pour cette raison, des indices particulièrement forts que des réformes sont en marche, en réaction aux problèmes des migrations.

Les dispositions prises pour permettre aux immigrés en Allemagne d'accéder à cette maîtrise de la langue ont été en général, comme l'étude le montre dans un premier temps, très caractéristiques : on les a traités comme des cas particuliers. Ceci est particulièrement net quand on considère les mesures prises pour les langues d'origine des immigrés.

16 I. Gogolin, M. Krüger-Potraz, «Beharrlichkeit und Innovation, Zwei Thesen zur Reaktion nationaler Bildungssysteme auf Migration und "Europäische Integration" », IZA 3/4, 1995, p. 74-79.

17 M. Krüger-Portratz, "Ein Blick in die Geschichte ausländischer Schüler und Schülerinnen in deutschen Schulen ", in : Éd. Ch. Kodron (et al.), Vergleichende Erziehungswissenschaft. Herausforderung - Vermittlung Praxis, Cologne, 1997, p. 656-672. 
Jusqu'au début des années quatre-vingt-dix, on a vu émerger les composantes fondamentales suivantes. Les immigrés nouvellement arrivés, ou les enfants dont les acquis (en allemand entre autres) étaient insuffisants pour qu'ils puissent entrer dans le système éducatif "régulier », furent dirigés provisoirement vers des classes d'accueil ou des classes "particulières", dans lesquelles l'enseignement pouvait aussi être assuré dans leur langue d'origine. Cette option leur était offerte dans le but déclaré de leur faciliter le passage vers le cursus "régulier ", donc dans la perspective d'une transition. L'enseignement de la langue dite maternelle devait faciliter l'acquisition de l'allemand deuxième langue. Parallèlement à cela, on trouvait dans certains Länder et pour des groupes sélectionnés d'immigrés, ce qu'on appelait un « enseignement complémentaire dans la langue maternelle » s'adressant à des enfants suivant un cursus "régulier ». Cet enseignement n'était assuré en principe que dans les langues officielles des États d'origine et non dans celles parlées dans les familles des enfants. Entre huit et quatorze langues yougoslaves pouvaient ainsi être regroupées, selon leur classification. Il n'y avait que quelques rares exceptions, par exemple l'enseignement en langue kurde pour des enfants venus de Turquie, et le plus souvent à partir d'initiatives privées. Une autre réaction aux langues introduites par les immigrés consista à accepter que leurs compétences soient évaluées et reconnues lors d'examens externes. Il était possible, et cette possibilité existe toujours - mais pratiquement pour les seuls enfants et adolescents d'origine turque -, de prendre comme option obligatoire l' « enseignement dans la langue maternelle » dans le second cycle.

Ces premières mesures visaient, d'une part, à rendre possible aux élèves leur réintégration dans le système éducatif de leur pays d'origine (dans le cadre de ce qu'on appelait, sur le plan politique, l'« option du retour ») qui correspondait aussi aux intérêts des États d'origine. Elles avaient, d'autre part, pour objectif de rendre plus facile le passage à la langue allemande voire, selon le cas, une meilleure adaptation aux exigences de l'enseignement allemand " régulier ». Ce n'est que dans les années quatre-vingt qu'un autre argument est apparu en faveur de telles options d'enseignement : on a mis l'accent sur leur fonction de socialisation.

Après 1990, on assiste à de nouvelles évolutions qui montrent que quelques-uns des paramètres fondamentaux sont en train de changer. Prenons, pour l'illustrer, l'exemple suivant : dans le Land de Berlin, en 1996, un paragraphe de la loi concernant l'enseignement consacré aux enfants immigrés fait l'objet d'une modification lourde de conséquences. Au lieu d'utiliser, comme on le faisait jusque-là, l'expression d' "enfants étrangers ", qui faisait de la nationalité des enfants le critère décisif, on emploie aujourd'hui, logiquement, dans le paragraphe en question, celle d' "élèves dont la langue d'origine n'est pas l'allemand ». De cette façon on ne se réfère plus, pour ces élèves, à leur statut de citoyen de l'État dont ils sont originaires, qu'il s'agisse de prendre des 
mesures pour les aider à progresser ou pour faire en sorte qu'ils ne soient pas pénalisés. Avoir modifié la loi de la sorte conduit dorénavant à chercher de nouveaux critères aussi bien pour admettre des élèves dans des options spécifiques, où l'on enseigne dans les langues des minorités, que pour définir ces langues elles-mêmes puisqu'il n'est plus possible de se référer au pays d'origine. Les paramètres traditionnels en usage dans le domaine de la maîtrise de la langue évoluent, comme on peut l'observer à la lumière de cet exemple. La terminologie des dispositions prises en faveur des immigrés est en cours d'adaptation. On n'observe pas seulement à Berlin cet abandon du critère de citoyenneté pour déterminer les modes d'accès aux options particulières dans le système éducatif. On a compris, au niveau de la politique éducative, que le fait de se référer simplement au principe de citoyenneté ne permettait plus de prendre en compte, de façon équitable, la situation multilinguale et multiculturelle en Allemagne. On réagit ainsi, et ce n'est pas sans importance, à une situation que les migrations ont rendue de plus en plus complexe. De plus en plus d'enfants dans les écoles allemandes sont issus de familles naturalisées ou binationales, donc de familles qui ne sont allemandes que de manière formelle. Dans plusieurs Länder, on peut observer des tendances qui en sont les conséquences logiques : des enfants détenteurs de passeports allemands doivent aussi avoir accès à l'enseignement des langues d'origine des migrants. Cette évolution présente un caractère innovant. On voit se dessiner une tendance à dissocier d'un côté l'idéologie, visant à obtenir une homogénéité interne de la société et, de l'autre, des tâches que le système éducatif doit de plus en plus remplir dans une société plurielle. Ainsi, l'hétérogénéité linguistique et culturelle de la population scolaire est une donnée pédagogique importante pour l'ensemble du système éducatif, au moins au niveau rhétorique. C'est une donnée devant laquelle l'école ne doit pas seulement réagir en prenant des dispositions particulières, pour une clientèle spécifique, mais qui doit aussi se refléter dans un système éducatif universel, ouvert à tous.

\section{***}

Comme l'ont montré ces différents exemples, les grands débats actuels sur l'éducation en Allemagne portent sur les dispositions concernant les contenus, les structures et l'organisation du système éducatif. Nombre de ces dispositions sont remises en question du fait des changements rapides que nous vivons aujourd'hui dans les domaines techniques, économiques, sociaux et culturels et on cherche à savoir si elles sont bien appropriées, si elles remplissent leur fonction, ou si de nouvelles solutions doivent être trouvées. Il reste à espérer que les évolutions qui se dessinent auront des effets bénéfiques sur les perspectives d'avenir des générations montantes, ainsi que sur leurs chances de mener la vie qu'ils désirent. 